\title{
An Improved Dual Vision System for Seam Guidance
}

\author{
Zhenbo Qin, Yingming Quan* and Guanxi Li \\ South China University of Technology, Guangzhou, Guangdong, China \\ ${ }^{*}$ Corresponding author
}

\begin{abstract}
High-speed fillet welding of container corrugated sheet requires high welding quality and welding speed, and visual monitoring technology has been introduced to achieve automatic welding. Aiming at the shortcomings of the photographing and the controlling on oblique stages of the welding seam, the existing dual vision system for seam monitoring was improved. The pose of the camera with respect to each stage of the welding seam can be fixed via adding a rotating mechanism so that the calculation of the offset between the molten pool and the welding seam can be simplified. The method of data fusion of the two vision systems was proposed to guide the movement of the welding torch simply and reliably. The simulation results show that this improved system can effectively make the welding torch move along the welding seam, and its performance meets the requirements of the welding accuracy and the welding speed.
\end{abstract}

Keywords-corrugated sheet; fillet welding; dual vision guiding; rotating mechanism; data fusion

\section{INTRODUCTION}

In container manufacturing, the way of the corrugated sheet welding with the base plate is usually adopted to increase the strength of the entire structure and to improve the mechanical properties. In baiting and assembly process, many factors may cause the non-uniform width and uncertain local direction of the seam to be weld of the base plate and the corrugated plate, so it is necessary to monitor the welding seam in real time and to plan the movement of the welding torch in welding process. Because the visual sensor has the advantages of abundant information, high accuracy, quick sampling and good feasibility, it is widely used in the monitoring of welding process. The use of the visual sensor includes shooting the laser line and directly shooting the molten pool [1]. The former (advanced vision) projects the laser line in front of the welding torch to avoid the arc interference, and the trajectory of the welding seam was obtained according to the shape and the position of the filmed laser line on the surface of the workpiece [2-3]. The latter (current vision) usually adds optical filter in front of the camera to avoid the arc interference, the offset between the molten pool and the welding seam was obtained from the image [4-6]. The reference [7] put a dual vision scheme for seam monitoring in which the two ways are synthetically used. The position of the front welding seam is obtained to plan the movement of the welding torch by monitoring the laser line image at one ripple period before the molten pool. The current width of the welding seam and the offset between the end of the welding torch and the center of the welding seam are obtained from the images of the molten pool region. The design is more effective than simply using advanced vision or current vision. However, in reference [7], the welding torch and the current camera only move along one direction without changing the oblique stages of the corrugated plate, so the images may not be complete and the calculation of the offset is complex on the oblique stages. In addition, the reference [7] did not refer to the monitored data fusion of the advanced and the current vision system or realization means of electromechanical control. It is improved in this paper.

\section{SUMmary OF THE EXISTING DUAL VISION SYSTEM}

The diagram of the dual vision system for seam monitoring is shown in FIGURE I.

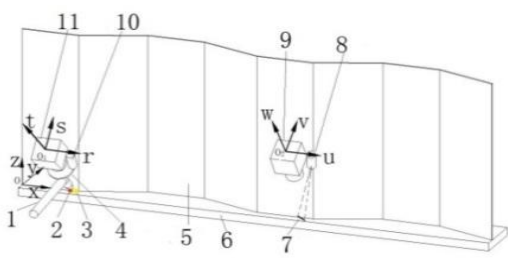

(a)3D view of the system

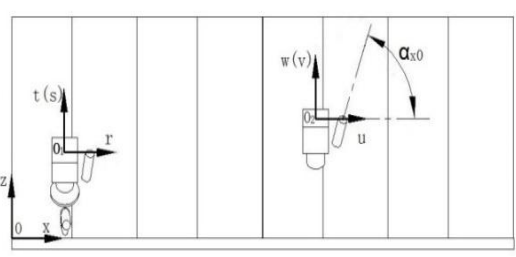

(c) Main view of the system

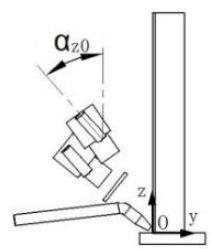
system

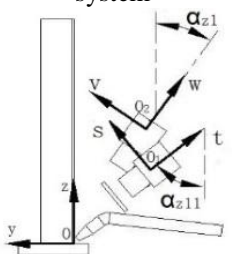

(d) Left view of the system (b) Right view of the

1.Welding torch; 2.Molten pool; 3.The spot; 4.The filter; 5 .Corrugated sheet 6.Base plate; 7.Laser line; 8.The laser; 9.Advanced camera; 10.Auxiliary light 11.Current camera

FIGURE I. STRUCTURE OF THE DUAL VISION SYSTEM FOR SEAM MONITORING

$\mathrm{O}-\mathrm{xyz}$ is the world coordinate system. The length direction of the corrugated plate is the positive direction of the $x$ axis; the height direction of corrugated sheet is along the $\mathrm{z}$ axis, the positive direction is from the bottom to the top, the $y$ axis is calculated by the right hand rule; the starting point of the welding seam is the original point.

$\mathrm{O}_{1}$-uvw is the advanced camera coordinate system. The $\mathrm{u}$ axis which is in the same direction as the $\mathrm{x}$ axis is parallel to the $x$ axis. The angle between the advanced camera optical axis $\mathrm{w}$ and the $\mathrm{z}$ axis is $\alpha_{\mathrm{z} 1}$ in the yz-plane. The angle between the laser and the $\mathrm{x}$ axis is $\alpha_{\mathrm{x} 0}$ in the $\mathrm{xz}$-plane. In the process of welding, the advanced camera moves along the $\mathrm{x}$ axis. 
$\mathrm{O}_{2}$-rst is the current camera coordinate system. The angle between the current camera optical axis $t$ and the $\mathrm{z}$ axis is $\alpha_{\mathrm{z} 11}$ in the yz-plane. The current camera is connected with the welding torch to move together. The distance between the advanced and the current camera is one ripple period. The $r$ axis which is in the same direction as the $\mathrm{x}$ axis is parallel to the $\mathrm{x}$ axis, when the welding torch is on the horizontal stages of the corrugated plate.

The images acquired respectively by the advanced and the current cameras are shown as FIGURE II (a), (b). In FIGURE II (b), the light point simulates the end of the welding torch.

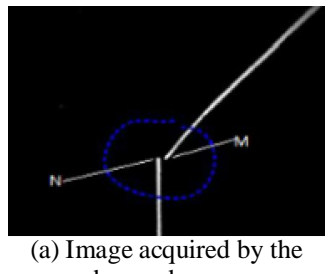
advanced camera

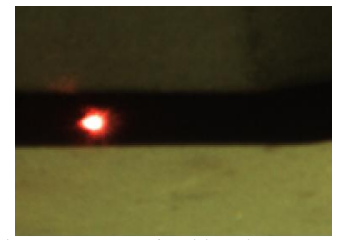

(b) Image acquired by the current camera
FIGURE II. IMAGES COLLECTED BY THE TWO SYSTEMS RESPECTIVELY

The trajectory of the corrugated sheet whose ripple period is $283 \mathrm{~mm}$ in $x y$-plane is shown in FIGURE III. The sheet includes stages (1), (2), (3) and (4).

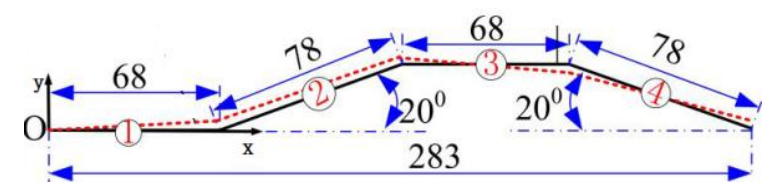

FIGURE III. THE TRAJECTORY OF THE CORRUGATED SHEET IN XY-PLANE

The principle of the dual vision system is shown in FIGURE IV. The trajectory of the welding seam is calculated according to the laser line image acquired by the advanced camera[8], and then it is transferred to the motion controller. The movement trajectory of the welding torch is designed quickly. Images of the molten pool and the welding seam acquired by the current camera are processed to get the shape and position of the molten pool, as well as the current position and the width of the welding seam. Assuming that the center of molten pool is equivalent to the position of the welding torch, the offset between the welding torch and the welding seam was obtained. The offset and the width are transferred to the welding process parameter adjuster and the motion controller to realize the dynamic compensation of the trajectory of the welding torch and welding parameters.

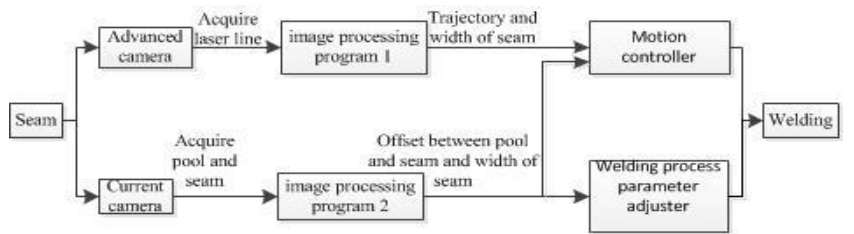

FIGURE IV. THE PRINCIPLE OF THE DUAL VISION SYSTEM

The advanced vision system only move along the $\mathrm{x}$ axis, so one motor which is noted as motor $\mathrm{x}$ is required. The movement of the current camera and the welding torch along the $\mathrm{x}$ axis are driven by the motor.

\section{THE IMPROVED DUAL VISION SYSTEM}

\section{A. The Addition of the Current Vision System of a Rotating Mechanism}

If the welding torch needs to move along 3D coordinates of the welding seam, then three motors are required. Besides, the welding torch shares the motor $\mathrm{x}$ with the advanced vision system, the current vision system, two more motors which are respectively denoted as motor $\mathrm{y}$ and motor $\mathrm{z}$ are needed to drive its movement along the $\mathrm{y}$ axis and the $\mathrm{z}$ axis. In reference [7], when the welding torch and the current camera reach the oblique stages of the corrugated sheet without rotation, the images of the molten pool and the conjoint welding seam may not be complete and the image coordinate system is hard to be transformed to the world coordinate system. Therefore, in the improved current vision system, when the welding torch and the current camera have arrived at the oblique stages (2), (4) of the corrugated plate, it should rotate to keep the pose with respect to the welding seam fixed, as it is on stages (1) and (3), so that photographing and calculation are simple and reliable. But one motor which is denoted as motor $r$ should be added to drive the rotation of the welding torch and the current camera. The movement velocity of motor $\mathrm{x}$ is controlled by the width of the welding seam measured by the current vision system. The motion of motor $y$ is controlled by y coordinates of the welding seam measured by the advanced vision system and the offset $\Delta y$ between the molten pool and the welding seam measured by the current vision system. Considering the gravity of the welding drop, the offset of the welding seam along $\mathrm{z}$ axis is ignored in actual welding process. The motion of motor $\mathrm{z}$ is only controlled by $\mathrm{z}$ coordinates of the seam measured by the advanced vision system. Motor $r$ is controlled by the theoretical trajectory of the welding seam.

The diagram of the welding torch rotating around the inflection point in xy plane is shown in FIGURE V. The rotating mechanism was designed by adopting the curved guide rail. The center of the guide rail is the end of the welding rod, and the radius of the curved rail is the length of the welding arm in xy plane. When the end of the welding torch is on the horizontal stages (1) and (3) of the corrugated sheet, the welding torch is in the center of the rail (Longitudinal symmetry). When the welding torch has reached the oblique stages (2) and (4) of the corrugated sheet, the current camera and the welding torch are driven by motor $r$ to rotate with required angle toward right or left. When the welding torch has reached the horizontal stages of the corrugated sheet, the current camera and the welding torch are driven by motor $r$ to rotate back the center of the rail. Therefore, the adjustment of the pose of the current camera and the welding torch can be realized. 


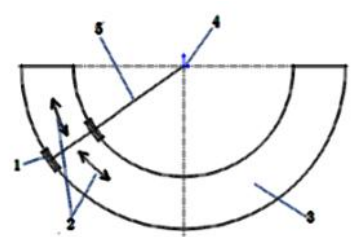

1. Slider; 2. Direction of welding torch motion; 3. Guide rail; 4. Point of inflexion; 5. Welding torch

FIGURE V. WELDING TORCH ROTATION DIAGRAM

In the improved dual vision system, the current camera coordinate system is $\mathrm{O}_{2}-\mathrm{r}^{\prime} \mathrm{s}^{\prime} \mathrm{t}^{\prime}$. The $\mathrm{r}^{\prime}$ axis is parallel to oblique stages of the corrugated plate. As shown in FIGURE VI.

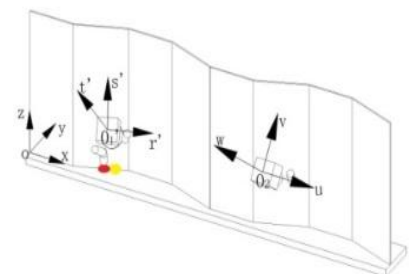

(a)3D view of the system

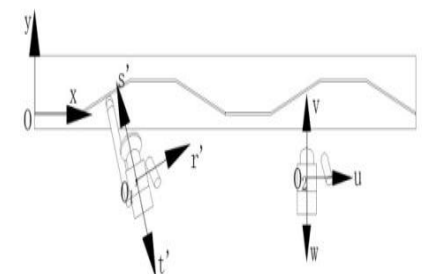

(b) Vertical view of the system
FIGURE VI. STRUTURE OF DUAL VISION SYSTEM FOR SEAM MONITORING WHEN THE WELDING TORCH IS ON THE OBLIQUE STAGES

\section{B. The Simplified Calculation of the Offset and the Width}

The current vision system is adopted to calculate the offset between the molten pool and the welding seam as well as the width of the welding seam. The imaging diagram of the welding process of stages (1) and (3) is shown in FIGURE VII (a). Here, points A, B, C and D represent the center of the molten pool, the low edge of the welding seam, the high edge of the welding seam and the center of the welding seam, respectively. The acquired images and calculation are shown in FIGURE VII (b). Here, A', B', $\mathrm{C}^{\prime}$ and $\mathrm{D}^{\prime}$ represent respectively the center of the welding pool, the low edge of the welding seam, the high edge of the welding seam and the center of the welding seam corresponding to FIGURE VII (a), and $\delta$ is the width of the welding seam in the image coordinate system.

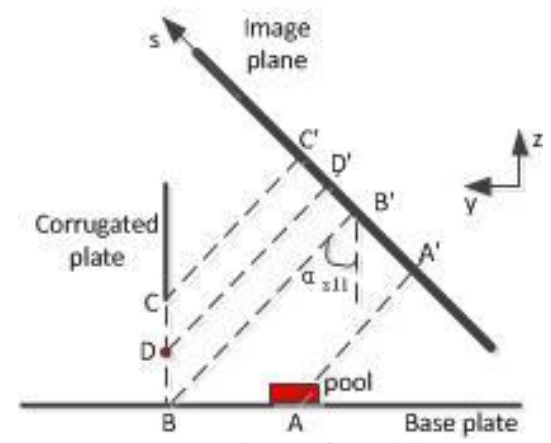

(a) Geometric model

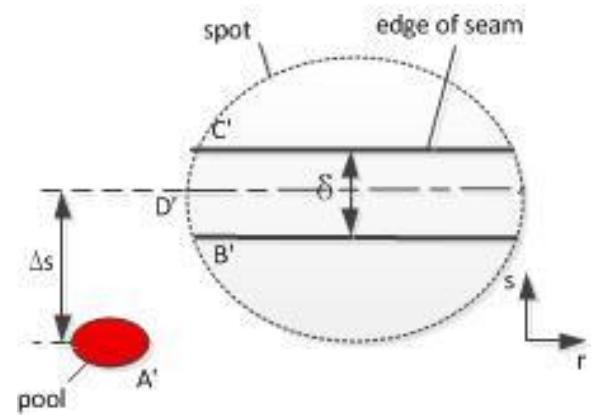

(b) Feature of image

FIGURE VII. ANALYSIS OF THE CURRENT VISION SYSTEM

If the distance between the center of the molten pool and the center of the welding seam along $s$ axis in rs coordinate system is $\Delta s$ (the distance between $\mathrm{A}^{\prime}$ and $\mathrm{D}^{\prime}$ ), then the offset of the center of the molten pool (the end of the welding torch) is [9]

$$
\Delta y_{1,3}=\lambda \cdot \Delta s \cdot \sin \left(90^{\circ}-\alpha_{z 11}\right)
$$

$$
L=\lambda \cdot \delta
$$

Here, $\lambda$ is the pixel equivalent of the current camera, $\Delta y_{1,3}$ is the offset on stages (1) and (3), $L$ is the width of the welding seam.

The current vision system rotates on the stages (2) and (4) so that the $r^{\prime}$ axis is parallel to the oblique stage, as shown in FIGURE VIII, so acquired images are identical with FIGURE VII (b). Then $\Delta y^{\prime}$ is obtained according to (1), and it is transformed to

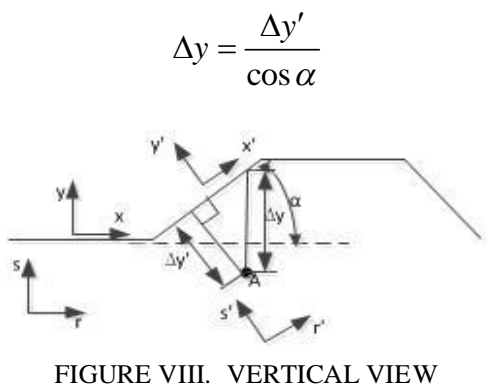

\section{Data Fusion of the Dual Vision System}

The advanced camera and the current camera can sample simultaneously by multithreading technology [10]. In actual welding system, the data measured by the two vision systems should be fused to guide the movement of the welding torch according to the model of the motion control. This paper adopted linear model to control the movement of the welding torch in xy-plane.

If the $\mathrm{x}$ coordinate of the advanced camera is $x_{a}$, then the measured coordinate of the welding seam is $\left(y_{a}, z_{a}\right)$. Because the advanced camera is in front of the current camera (one ripple period, $283 \mathrm{~mm}$ ), now the coordinate of the current 
camera is $x_{a}-283 \mathrm{~mm}$. When the $\mathrm{x}$ coordinate of the current camera is $x_{a}$, the offset of the molten pool should fuse with the coordinate of the welding seam measured by the advanced vision system here, i.e., adding $\Delta y$ according to $y_{a}$.

$$
d_{y}=y_{a}+\Delta y
$$

Here, $d_{y}$ is the required displacement of the welding torch along y axis, i.e., the control value of the motor $y$.

\section{The Simulation OF WeLDING EXPERIMENT}

The simulation experiment which the welding torch was replaced by a laser pen was designed, and the molten pool was replaced by a laser spot. Before welding, the corrugated sheet and the base plate should meet the following assembly relations. The corrugated sheet connects closely with the base plate, and the width of the gap between the bottom of the corrugated sheet and the base plate along $\mathrm{z}$ axis is gradually increasing along $\mathrm{x}$ axis.

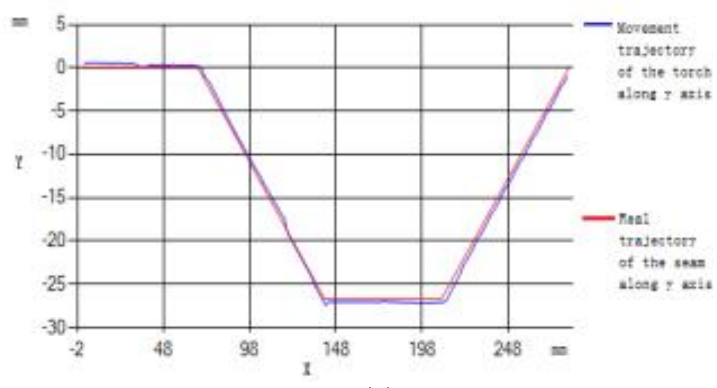

(a)

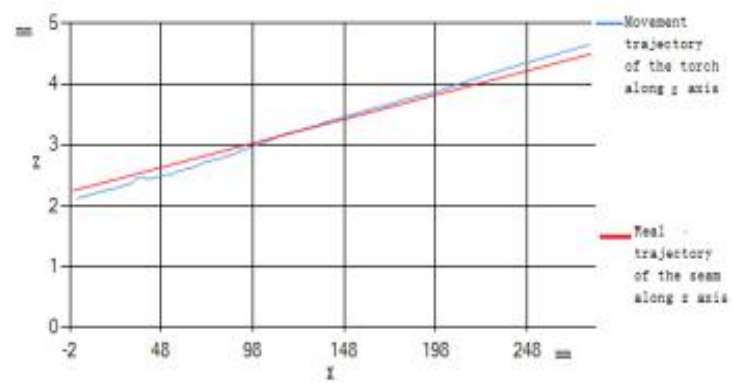

(b)

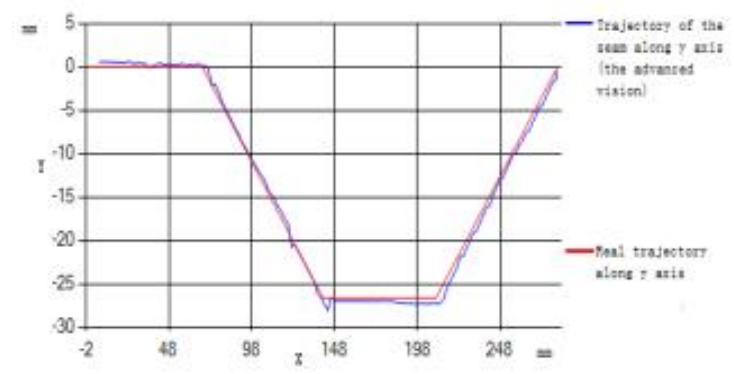

(c)

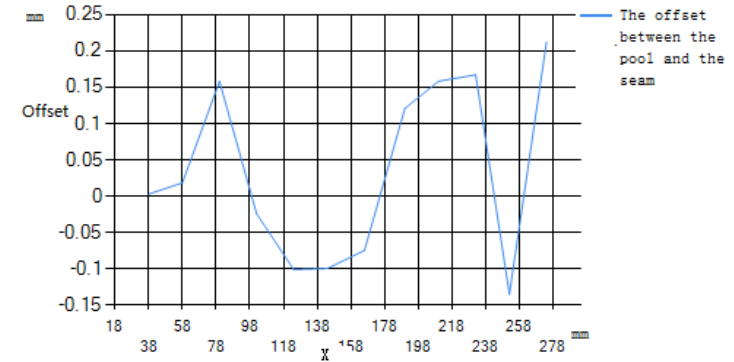

(d)

FIGURE IX. THE MOVEMENT OF THE WELDING TORCH

In FIGURE XI (a) and (b), the red line represents respectively the real trajectory of the seam in xy- and xz-plane, the blue line represents respectively the movement trajectory of the end of the welding torch in xy-plane and xz-plane. It can be seen from the two graphs, that the blue line and red line basically coincide, i.e., the end of the welding torch moves accutely along the trajectory of the welding seam. In FIGURE XI (c), the red line still represents the actual trajectory of the welding seam in the xy-plane, and the blue line represents the trajectory of the end of the welding torch in the xy-plane which is measured by the advancd vision system. The fact that both lines do not coincide illustrates that the accuracy is not high when the advanced vision system is only adoped to guide the movement of the end of the welding torch along the actual trajectory of the welding torch. As shown in FIGURE XI (d), there is the offset between the molten pool and the welding seam measured by current vision system in whole welding process. So the data fusion of dual vision system for the guidance of the welding torch is more accurate.

\section{CONCLUSIONS}

Adding a rotating mechanism to keep the pose of the camera with respect to the welding seam fixed, the calculation of the width of the welding seam as well as the offset between the center of the welding seam and the welding torch is simplied. A method of the data fusion of the dual vision system was proposed to control the motor. The simulation results show that there is a large error with respect to the actual welding seam when using only the advanced vision system to guide the movement of the welding torch, and the use of the monitored data fusion of the dual vision system can reduce the guidance error of the welding torch.

\section{ACKNOWLEDGMENT}

This work is supported by the key project of the strategic cooperation among Guangdong Province and Chinese Academy of Science, 2013(2013B091000006).

\section{REFERENCES}

[1] GUO Bo, SHI Yong-hua, "Vision-Based Welding Deviation Detection for CO2 Arc Welding," ournal of South China University of Technology(Natural Science Edition), vol. 44, pp. 1-8, 2016.

[2] Noruk J, "Visual weld inspection enters the new millennium," Sensor Review, vol.21, pp.278-282, 2001. 
[3] Lee J, Im P, Park Y, et al. "Welding bead and chamfer inspection by means of laser vision," Optomechatronic Systems 2000, Proceedings of SPIE, vol.4096, pp. 1-6, 2001.

[4] Gao X, et al. "Infrared image recognition for seam tracking monitoring during fiber laser welding," Mechatronics, vol.22, pp.370-380, 2011.

[5] Shi Y H, Wang G R, "Vision based seam tracking system for underwater flux cored arc welding," Science and Technology of Welding and Joining, vol.11, pp. 271-277, 2006.

[6] YE ZH, FANG G, CHEN S B,et al. "Passive vision based bean tracking system for pulse-MAG welding," The International Journal of advanced Manufacturing Technology, vol.67, pp.1987-1996, 2013

[7] Bi Qinlin. "Dual Visual Tracking and Monitoring of Large Structure's Ripple Line Fillet Seams Based on Advanced and Current." Dissertatioin. South China University of Technology, 2014.

[8] Yanming Quan, Qilin Bi. "Tracking and Monitoring of 3-Dimensions of Welding Seam and Width in Fillet Welding of Corrugated Sheet." International Journal of Control and Automation, vol.8, pp.337-350, 2015.

[9] Bi, Qi Lin, Y. M. Quan. "Ripple Line Fillet Seam Tracking with Visual Sensors in Welding Process." Key Engineering Materials, vol.579-580, pp.46-51, 2014

[10] XU Yi,FENG Shan," Multithreaded Programming by Using C\#," Computer Engineering, vol.29, pp.1-3, April 2003 